\title{
Modalidad educativa de los colegios de procedencia de jóvenes con discapacidad cognitiva que asisten a un programa de inclusión en la educación superior*
}

\author{
Lucía Illanes Aguilar*, María Theresa von Furstenberg Letelier ${ }^{\star \star \star}$
}

RESUMEN

El estudio describe los colegios de procedencia según su modalidad educativa de alumnos con discapacidad cognitiva que asisten a un programa de inclusión en la Educación Superior. Es un estudio cuantitativo, descriptivo y longitudinal y se concluye que, entre el 2006 y el 20I4, los jóvenes con Necesidades Educativas Especiales (NEE), que se han capacitado para el mundo laboral en este programa universitario, finalizaron la Educación Media en establecimientos que no contaban con Proyecto de Integración Escolar (PIE).

Palabras clave: Modalidad educativa, inclusión, Educación Superior.

Schools of origin of young people living with cognitive disabilities and attending inclusion programs in Higher Education

\section{ABSTRACT}

The study describes the schools of origin according to their educational modality of students with cognitive disabilities attending an inclusion program in Higher Education. It is a quantitative, descriptive and longitudinal study and it was concluded that, between 2006 and 20I4, young people with special educational needs (SEN), who have qualified for the world of work in this university program, completed Secondary Education in establishments lacked Project School Integration (PIE).

Keywords: Educational methods, inclusion, Higher Education.

* Este artículo forma parte de un estudio de Doctorado en Psicología de la Facultad de Psicología y Educación de la Pontificia Universidad Católica Argentina.

* Lucía Illanes Aguilar es Doctora en Nuevas Perspectivas en Investigación en Ciencias de la Actividad Física y el Deporte de la Universidad de Granada, Magíster en Administración y Gestión Educacional de la Universidad Mayor, Post Título en Administrador Educacional de la Universidad Católica del Norte, Profesora de Educación Física, Pontificia Universidad Católica de Valparaíso. Profesora Asociada de la Facultad de Humanidades de la Universidad Andrés Bello (UNAB). E-mail: lillanes@unab.cl

María Theresa von Furstenberg Letelier es Candidata a Doctora en Psicología de la Universidad Católica de Buenos Aires, Magíster en Recursos Humanos del Instituto de Directivos de Empresas, CESEM España, Psicóloga de la Universidad de Chile. Directora del Programa en Habilidades Laborales Específicas de la Facultad de Humanidades de la Universidad Andrés Bello (UNAB). E-mail: mvonfurstenberg@unab.cl 


\section{Introducción}

De acuerdo a la Encuesta Nacional de Discapacidad (MINEDUC, 2004) en Chile, aproximadamente, el 12,9\% de la población tiene algún tipo de discapacidad. De ellos, solo $25 \%$ ingresa al sistema formal de enseñanza y un 6,6\% a la Educación Superior (FONADIS, 2004).

Las modalidades educativas que se ofrecen en el país para satisfacer las Necesidades Educativas Especiales (NEE) aún se clasifican y se describen de acuerdo a lo que establece la Ley de Integración Social de las Personas con Discapacidad No I9.284 (Gobierno de Chile, I994), modificada por la Ley 20.422 en el año 2010 (SENADIS, 20I0).

Según el Decreto I98 de Educación Especial en su Artículo I ${ }^{\circ}$, el sistema escolar chileno en su totalidad deberá entregar alternativas educacionales a los estudiantes que presenten NEE, pudiendo hacerlo a través de establecimientos comunes de enseñanza con proyectos de integración y/o las escuelas especiales.

\section{Marco teórico}

\subsection{Modalidad educativa}

Desde 1994, el Ministerio de Educación de Chile (MINEDUC) responde a las nuevas normativas con la Política de Integración Escolar (PIE). De acuerdo a esto, se pueden definir las siguientes modalidades educativas:

En primer lugar, están aquellos colegios que integran a alumnos con discapacidad o trastornos específicos del lenguaje, es decir, aquellos que deben generar un PIE, que es una estrategia inclusiva para el mejoramiento continuo de la calidad de la educación en el establecimiento educacional. Su propósito es garantizar la presencia en la sala de clases y la participación, favoreciendo el logro de los objetivos de aprendizaje de "todos y cada uno de los estudiantes", 
especialmente, de aquellos que presentan NEE, sean estas de carácter permanente o transitorio (MINEDUC, 2013).

Para ellos se establecen las siguientes acciones a realizar:

I. Coordinación de los PIE al interior del establecimiento.

2. Contratación de profesionales asistentes de la educación, de acuerdo a las NEE de los/as estudiantes.

3. Establecer estrategias de trabajo colaborativo y de co-enseñanza.

4. Aplicación de adecuaciones curriculares y apoyos especializados dirigidos a los/as estudiantes que presentan NEE, de acuerdo a la Evaluación Diagnóstica Integral.

5. Contar con un "Aula de recursos".

6. Acciones tendientes a informar del PIE a la comunidad educativa y, especialmente, a las familias de los estudiantes del establecimiento, a ellos mismos cuando corresponda y considerar sus intereses.

7. Capacitación y perfeccionamiento sistemático y orientado al desarrollo profesional de los docentes de educación regular y especial y a otros miembros de la comunidad educativa.

Es importante añadir que los PIE permiten que alumnos con NEE sean parte de cualquier tipo de establecimiento educativo, sean estos municipalizados, particulares subvencionados con o sin financiamiento compartido y particulares pagados.

En segundo lugar, se encuentran los establecimientos de educación regular con grupos diferenciales, que buscan apoyar el proceso educativo de alumnos con NEE de la Educación Básica que presentan dificultades de aprendizaje y adaptación escolar (MINEDUC, 20I3), los cuales no están plenamente incluidos en las aulas regulares, pero sí en la institución educacional regular.

En tercer lugar, están las escuelas especiales. Se trata de una modalidad del sistema escolar que provee servicios y recursos especializados, tanto a los establecimientos de enseñanza regular como a las escuelas especiales, con el propósito de asegurar, de acuerdo a la normativa vigente, aprendizajes de calidad a niños, 
niñas y jóvenes con necesidades educativas especiales asociadas o no a una discapacidad, asegurando el cumplimiento del principio de igualdad de oportunidades para todos los educandos. (Ministerio de Desarrollo Social, 2015).

En cuarto lugar, están las escuelas y aulas hospitalarias, que ofrecen educación compensatoria a alumnos que cursan únicamente la Educación Básica, de tipo regular y especial, que por dificultades de salud deben permanecer en centros hospitalarios o en tratamiento ambulatorio durante un periodo continuado, que como rango mínimo alcanza los tres meses. A la fecha existen 34 aulas de esta modalidad, que atienden a alrededor de I.200 alumnos (MINEDUC, 20I3).

Por último, si se habla de modalidades educativas no se puede dejar de mencionar a los establecimientos comunes de enseñanza que no cuentan con un programa de apoyo formal que es aprobado como tal por el Ministerio de Educación, pero que, sin embargo, cuentan con profesionales, tales como psicólogos, educadores diferenciales o psicopedagogos que apoyan el proceso educativo de alumnos que presentan NEE.

En esta modalidad educativa no hay forma de evaluar el logro de los objetivos de aprendizaje, ya que estos se evalúan internamente por el establecimiento educacional y no hay parámetros de comparación entre un establecimiento y otro.

El sistema educacional faculta a los jóvenes que asisten a estos programas para integrarse a la Educación Básica y media regular mediante los ya mencionados PIE, no obstante, esta modalidad educativa no tiene una continuidad natural en la Educación Superior. Por lo tanto, los jóvenes con necesidades educativas especiales por discapacidad cognitiva deben volver a ambientes segregados con el propósito de formarse para un futuro laboral.

El programa de formación socio-laboral "Diploma en habilidades laborales" implementado por la Universidad Andrés Bello el año 2006, seguido por la Universidades Central y, recientemente, la Universidad de Los Lagos, responden a esta falencia del sistema educacional inclusivo para estos jóvenes. 
Según la Ley 20.422 "las instituciones de educación superior deberán contar con mecanismos que faciliten el acceso a las personas con alguna discapacidad, así como adaptar los materiales de estudio y medios de enseñanza para que dichas personas puedan cursar las diferentes carreras" (SENADIS, 2010, p. 2I). Sin embargo, esto no aplica para los jóvenes con discapacidad intelectual, sino a los jóvenes con discapacidad sensorial o motora, lo cual implica adaptar los medios de acceso al conocimiento, no la profundidad ni naturaleza de los mismos.

\subsection{Tipo de establecimiento educativo}

Otra variable importante de analizar en este artículo corresponde al tipo de colegio, entendiendo por ello el tipo de financiamiento $y$, por lo tanto, el nivel socioeconómico asociado, que podría estar afectando al desarrollo de la autonomía en cada caso.

Es importante destacar que en el país la Educación Básica y Media son obligatorias, debiendo el Estado financiar un sistema gratuito en función de asegurar el acceso a ellas de toda la población, así como crear las condiciones para la permanencia en el mismo, según lo que dicta la ley. Al respecto, se indican los siguientes:

- Modalidad pública o municipalizada: cuando la educación es gratuita o municipalizada el ente financista es el Estado, el cual entrega la totalidad de los recursos para el funcionamiento de los establecimientos educativos. Estos, a su vez, son administrados por los municipios o gobiernos locales.

- Modalidad particular subvencionada: los sostenedores privados en el sector subvencionado reciben aportes del Estado para financiar la operación de sus establecimientos y recursos adicionales a través del régimen de financiamiento compartido, atendiendo al 46,8\% del total de la población escolar. Al contrario de las escuelas municipales y los colegios particulares pagados, los sostenedores particulares subvencionados presentan heterogeneidad en cuanto a su distribución geográfica (el $77 \%$ de las comunas tienen, a lo menos, un colegio particular subvencionado y estos atienden al 22\% del total de la matrícula 
básica rural), composición social de los estudiantes atendidos en sus establecimientos (casi el $20 \%$ de sus alumnos son prioritarios, dos tercios de la clase media y I4\% de los alumnos categorizados en la categoría más alta del MINEDUC provienen del sector particular subvencionado), que se manifiesta, además, en las distintas personalidades jurídicas (con y sin fines de lucro), escalas de operación, misiones declaradas, y religiosidad. Hasta la fecha, pocos autores han explorado la diversidad del sector (Brunner y Elacqua, 2004).

La proporción de estudiantes en establecimientos particulares subvencionados ha venido aumentando sostenidamente desde el año I980; en 1990 alcanzó un 32\%, un 36\% en 2000 y más del 45\% en 2005. Este crecimiento del sector particular subvencionado se produjo, principalmente, por una progresiva reducción del sector municipal en el total de la matrícula, que cae del $78 \%$ a un $45 \%$, considerando el periodo I98I-2008 (Corvalán, Elacqua y Salazar, 2009).

- Modalidad particular pagada: estos colegios se establecen por personas, sociedades o corporaciones que administran sus propios recursos, se financian íntegramente con el aporte de apoderados o de entidades. Al no existir aportes del Estado, el control de este es escaso; no obstante, el MINEDUC entrega documentos orientadores para la gestión de la enseñanza en el sistema escolar.

La matrícula en establecimientos particulares pagados, cercana al $7 \%$, se mantiene estable a lo largo del tiempo. En suma, actualmente más de la mitad de los alumnos de Enseñanza Básica y Media son atendidos por establecimientos privados, sean estos subvencionados o particulares pagados.

Los colegios particulares pagados -que no reciben subvención por alumno- controlan y atienden a un porcentaje muy pequeño de la población escolar (7\%), están concentrados en pocas comunas ( 3 de cada 4 comunas no tienen colegios pagados y el $26 \%$ de su matrícula básica está en Las Condes, Vitacura y Providencia), casi todos se localizan en zonas urbanas (97\%), no atienden alumnos prioritarios y sus estudiantes son del estrato socioeconómico más alto. 
Por el contrario, la educación municipalizada y particular subvencionada es ampliamente regulada por el Estado, pues la financia de manera parcial o total a través de recursos públicos. La Superintendencia de Educación, también, es un ente regulador de la educación municipalizada y particular subvencionada, dado que posee las atribuciones de fiscalizar y sancionar el cumplimiento de la normativa, fiscalizar los recursos y auditar rendición de cuentas, investigar y resolver denuncias y hacer de mediador con los reclamos y entregar información de interés a la comunidad escolar.

\subsection{Conceptualización de inclusión educativa y su presencia en Chile}

La inclusión educativa es entendida por la UNESCO (2008) como un proceso en el que se minimizan las barreras al aprendizaje, abriendo la participación de todas las personas al sistema educativo. Si se habla de educación inclusiva es necesario hacer mención a la integración, la que es comprendida, por esta misma institución, como la adecuación de la persona al espacio educacional, sin focalizarse en las modificaciones del entorno, eliminando barreras (UNESCO, 2008).

Basado en el concepto de atención a la diversidad, consolidado en la Conferencia Mundial sobre Necesidades Especiales (UNESCO, I994), se comienza a pensar en la integración escolar, refiriéndose al acceso físico de personas con discapacidad a ambientes que habían sido exclusivos para personas sin discapacidad, sin que -en muchos casos- en este ambiente se realicen los ajustes para responder a las diversas necesidades de los estudiantes, evitando obstáculos y/o barreras en sus procesos de aprendizaje.

La educación inclusiva, en cambio, busca asegurar el derecho a la educación a todos los estudiantes, considerando todas sus características y dificultades individuales, permitiéndoles no solo el acceso a estos espacios, sino que también la participación plena en igualdad de oportunidades con sus pares (Lissi et al., 20I3). De esta forma, la perspectiva de la educación inclusiva se basa en una modificación profunda en la manera de entender la discapacidad. 
Esto implica que es el sistema educativo el que incluye y se estructura para satisfacer la necesidad de los estudiantes (Stainback, Stainback y Jackson, 1999) y, por lo tanto, es en dicho sistema que recae la responsabilidad de hacerlo y no, como sería desde la perspectiva de la integración, el alumno quien debe integrarse. Así, la perspectiva inclusiva estaría asociada al Modelo Social de la Discapacidad, pues se focaliza en aquellos aspectos ambientales y de interacción con el contexto, más que en aspectos relativos al déficit (Moreno, 2005).

Por otro lado, es relevante señalar que el concepto de NEE comenzó a ser utilizado en los años setenta. El Informe Warnok, encargado por la Secretaria de Educación del Reino Unido en I978, facilitó la incorporación de esta nueva concepción a la práctica educativa (Lucchini, 2003).

De acuerdo con Garrido y Santana (I994) las NEE se presentan cuando una deficiencia física, sensorial, intelectual, social o cualquier combinación de estas afectan el aprendizaje, requiriendo de modificaciones al currículum ya sean parciales o totales.

Las NEE pueden presentarse de manera permanente o transitoria en el desarrollo del alumno, entendiéndose por transitorias aquellas en las que los alumnos requieren ayudas y recursos solo en un momento determinado de su escolarización; otros, en cambio, requieren de estos apoyos en forma sostenida en el tiempo; estas son las que se asocian a discapacidad.

Según Lucchini (2003) cuando las NEE están ligadas a una discapacidad de tipo cognitivo, aunque esta sea leve, los alumnos no lograrán los mismos aprendizajes que otros niños o jóvenes de su edad que no muestren discapacidad, pero desarrollarán el máximo de su propio potencial.

Junto a la incorporación del concepto de NEE, en los últimos años se han trazado caminos y estrategias para reducir la exclusión y aumentar el acceso y la participación en el sistema regular de enseñanza de estudiantes que presentan discapacidad (Eitel, 2005).

La integración y/o inclusión educativa realizada en las debidas condiciones es positiva para los alumnos con NEE, ya que contribuye 
a su mejor desarrollo y a una más completa y normal socialización (Blanco, Ríos y Benavides, 2004). Además, es beneficioso para el conjunto de alumnos, pues todos aprenden con una metodología más individualizada, más atenta a la diversidad de situaciones en las que se encuentran. La integración, finalmente, desarrolla en los alumnos actitudes de respeto y de solidaridad hacia sus compañeros con mayores dificultades, lo que es uno de los objetivos relevantes de la educación (Marchesi y Coll, I998).

Para hacer una distinción entre la incorporación de niños con NEE a la escuela y la educación adaptada a la diversidad del alumnado, en los últimos años se ha empezado a hablar de inclusión educativa. Se entiende el concepto de inclusión como más amplio que el de integración, dado que intenta entregar modalidades de trabajo educativo que minimicen la necesidad de las escuelas segregadas y mejoren la capacidad de la escuela regular de responder a la diversidad (Ainscow, 200I; Blanco, 20II y Mertz, 200I).

Frente a la discusión respecto a la utilización de los términos integración o inclusión, algunos autores señalan que dicha discusión es "simplemente nominalista, pues hay una tendencia a sustituir el término integración educativa por el de inclusión educativa para resaltar los productos de los últimos debates y reflexiones sobre la educación en y para la diversidad" (Verdugo, 2003, p. II).

En la última década, las instituciones de Educación Superior han gestionado en favor de la paulatina incorporación de los estudiantes con NEE. Sin embargo, la dificultad que entraña la inclusión de personas con discapacidad intelectual en un entorno universitario ha contribuido a que, hasta el momento, no se hubiesen atendido realmente las necesidades de este colectivo (Izuzquiza y Rodríguez, 2010).

En Chile, la matrícula de los estudiantes con distintas NEE en el sistema escolar ha crecido de manera importante. Los estudiantes con NEE transitorias han aumentado en un $1.250 \%$ y aquellos con NEE permanentes, especialmente, discapacidad intelectual han duplicado su número (Centro de Políticas Comparadas en Educación, 2015). No así la incorporación de ellos a la Educación Superior. 
Las escuelas especiales o diferenciales constituyen servicios educativos con una gran trayectoria en nuestro país. Estos establecimientos pueden ser reconocidos oficialmente por el Estado y recibir subvención escolar. Los centros de educación especial atienden a estudiantes con NEE permanentes o NEE transitorias entre los 2 y 24 años de edad cronológica. Cabe mencionar que estas escuelas cuentan con planes y programas de estudio específicos por tipo de discapacidad, los que en su mayoría fueron aprobados en los años '90 por el MINEDUC. Es decir, los estudiantes que asisten a estos establecimientos educacionales cuentan con un currículo paralelo y distinto al currículo nacional, lo que ha sido descrito como una barrera importante a la hora de generar procesos de inclusión educativa (Duk y Loren, 2010).

Concretamente, en el ámbito de la Educación Especial el tipo de formación laboral para los jóvenes con discapacidad intelectual es realizada en los talleres laborales, los que aparecen estipulados en el Decreto No 87/I990 del MINEDUC y que tienen entre sus propósitos proporcionar formación laboral, a través del desarrollo de habilidades, considerando intereses de los estudiantes, así como también los requerimientos sociales, sin embargo, "el sistema institucional chileno no logra responder adecuadamente a las demandas de las personas con discapacidad intelectual en el acceso y permanencia en el trabajo competitivo" (Vidal y Cornejo, 2012, p. 99).

Al profundizar en la población más afectada, se ha demostrado que entre los egresados de los establecimientos educacionales que forman laboralmente a los jóvenes que presentan discapacidad, la mayoría no logra insertarse adecuadamente al mercado laboral y los empleadores, a su vez, no encuentran en los postulantes los perfiles de calificación requeridos. La formación en ambientes segregados no facilita la adquisición de las habilidades sociales necesarias para una completa inserción social (Bravo, Peirano, Sevilla y Weintraub, 200I).

Como se explicitó anteriormente, en Chile, durante las últimas décadas se ha producido un aumento de jóvenes con NEE por 
discapacidad intelectual que ha finalizado su educación media en colegios regulares con modalidad de educación inclusiva. Esta realidad se vio ligada a dos variables importantes:

a. La imposibilidad de que estos jóvenes puedan acceder a la Educación Superior en forma normalizada, aunque se realicen las adaptaciones necesarias que señala la ley, y,

b. La reticencia de las familias a que los jóvenes vuelvan a la educación segregada para su formación laboral generó una necesidad educacional, que motivó la implementación en la Universidad Andrés Bello de un programa de inclusión educacional a la Educación Superior.

\section{Metodología}

El estudio es de tipo cuantitativo y su alcance es descriptivo. Además, presenta un diseño no experimental y su corte es longitudinal.

La población del estudio la constituyen 288 estudiantes pertenecientes al Programa. La muestra está constituida por 2I3 estudiantes de las promociones 2006 a 2013. El muestreo es no probabilístico, por conveniencia.

Por último, en el estudio se utilizan fichas de registro de datos para recabar la información.

\section{Resultados}

Los estudiantes del Programa pertenecientes a las promociones 2006 a 2013 en la sede de Santiago suman 2 I3 jóvenes; su distribución, considerando la modalidad educativa, es la siguiente:

- Colegios regulares con PIE I2,7\%.

- Colegios regulares sin PIE 4I,3\%.

- Colegios regulares definidos para NEE 28,6\%.

- Escuelas especiales 17,4\%. 


\section{Gráfico I. Modalidad educativa}

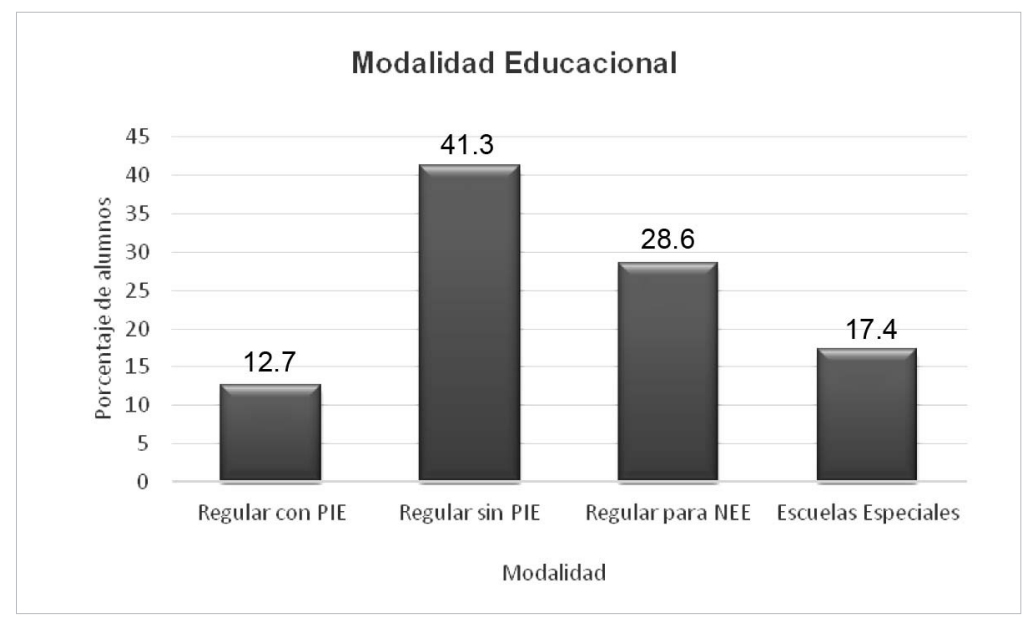

Fuente: Elaboración propia.

En relación al tipo de colegio, la muestra se distribuye en:

- Colegio Particular Pagado: $70 \%$

- Colegio Particular subvencionado: $22 \%$

- Colegio Municipalizado: 8\%

Gráfico 2. Tipo de dependencia

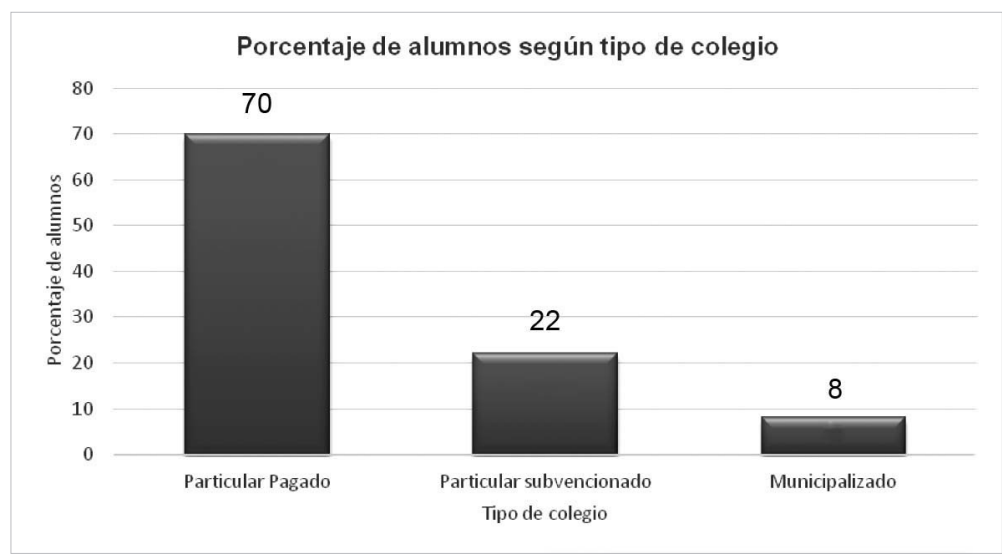

Fuente: Elaboración propia. 
En cuanto a las comunas de procedencia de los estudiantes del Programa, estos son de las siguientes comunas:

Tabla I. Distribución comunas de procedencia

\begin{tabular}{|l|c|l|c|}
\hline \multicolumn{1}{|c|}{ COMUNA } & $\begin{array}{c}\mathrm{N}^{\circ} \\
\text { ALUMNOS }\end{array}$ & \multicolumn{1}{c|}{ COMUNA } & $\begin{array}{c}\mathrm{N}^{\circ} \\
\text { ALUMNOS }\end{array}$ \\
\hline Las Condes & 77 & Huechuraba & 2 \\
\hline Lo Barnechea & 40 & Independencia & 2 \\
\hline Vitacura & 33 & La Florida & 2 \\
\hline Providencia & I6 & Paine & 2 \\
\hline La Reina & II & Cerrillos & I \\
\hline Nuñoa & 4 & La Cisterna & I \\
\hline Macul & 3 & Pirque & I \\
\hline Maipú & 3 & San Miguel & I \\
\hline Peñalolén & 3 & Talagante & I \\
\hline San Bernardo & 3 & Buin & 2 \\
\hline Santiago & 3 & & \\
\hline Calera de Tango & 2 & & \\
\hline
\end{tabular}

Fuente: Elaboración propia.

Gráfico 3. Comunas de procedencia de la muestra

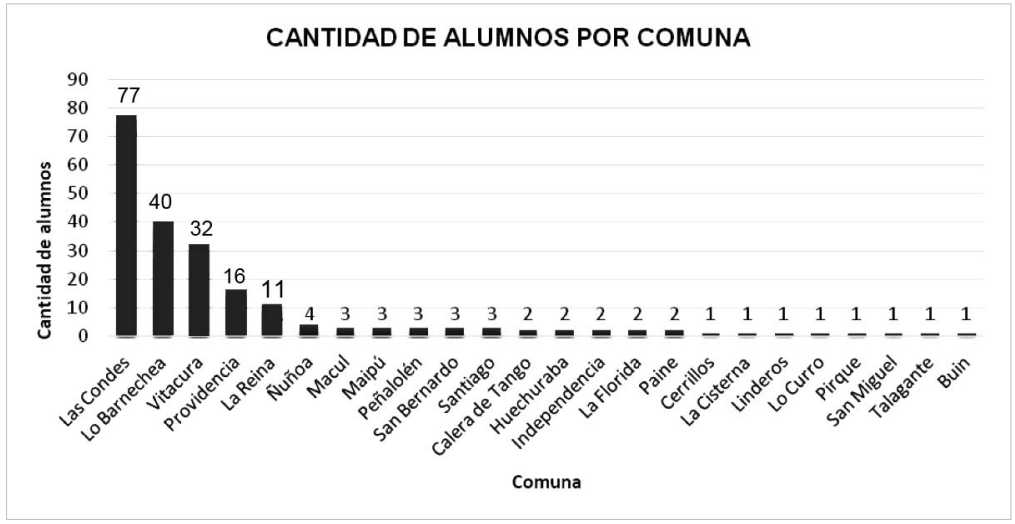

Fuente: Elaboración propia. 


\section{Conclusiones}

Todos los jóvenes de la muestra presentan discapacidad cognitiva o intelectual leve, situación que indicaría que los sujetos debieran provenir de colegios de educación regular con PIE, no obstante, la inclusión con esta modalidad educacional no ha sido una alternativa para ellos.

Si se analizan los colegios de procedencia, es posible observar que solo un $\mathrm{I} 2,7 \%$ procede de colegios con PIE oficiales y un $17,4 \%$ de Educación Especial. El 69,9\% restante se distribuye entre colegios regulares sin PIE y colegios regulares que se han definido a priori como colegios personalizados para niños con NEE.

Los colegios regulares tradicionales no acceden a PIE oficiales, sino que realizan en forma interna las adaptaciones curriculares para cada niño con NEE a través de sus equipos de profesionales. En esta condición se encuentra el 4I,3\% de los sujetos muestrales, cifra que permite inferir que poseer un PIE no es considerado como una alternativa a implementar por los colegios regulares que no se definen como personalizados, sino que optan por realizar las adaptaciones curriculares de manera informal.

Los colegios que se definen como personalizados, es decir, que tienen a la mayoría de sus alumnos con NEE no realizan PIE individuales ni adaptaciones curriculares para cada niño, sino que implementan un currículo universal que les permite trabajar con la diversidad del alumnado.

Un 17,4\% de los alumnos provienen de la Educación Especial. Estos jóvenes no se beneficiaron de la educación inclusiva durante las etapas previas de su proceso educativo, Educación Básica y Media, por lo cual para ellos esta experiencia educacional incluida en un ámbito universitario es su primera experiencia de inclusión y, por lo tanto, un desafío de adaptación.

Si se analiza el tipo de colegio, se puede observar la forma en que estos se distribuyen de acuerdo a su modalidad educativa. Es así como el $70 \%$ corresponden a colegios particulares pagados, pudiendo inferir 
que han permitido la inclusión educacional en los niveles básicos y medios y han aportado una mejor respuesta a las necesidades educativas especiales de los sujetos de la muestra.

Según las comunas de procedencia de los alumnos del Programa, estos se concentran, mayoritariamente, en la zona oriente de Santiago, comunas de Las Condes y Lo Barnechea, situación posible de explicar por la ubicación del Campus. ${ }^{\text {I }}$

\section{Referencias bibliográficas}

Ainscow, M. (200I). Desarrollo de escuelas inclusivas. Madrid: Narcea.

Blanco, R. (201 I). Educación inclusiva en América Latina y el Caribe. Revista Participación Educativa: Todos Iguales Diferentes, (I8), 46-59.

Blanco, R., Ríos, C. \& Benavides, M. (2004). Respuesta educativa para niños con talento. Santiago: Ediciones UNESCO.

Brunner, J. J. \& Elacqua, G. (2004). Factores que inciden en una educación efectiva. Evidencia internacional. Revista Virtual La Educación, I39-I40, I-II.

Corvalán, J., Elaqua, G. \& Salazar, I. (2009). El sector particular subvencionado en Chile. Tipologización y perspectivas frente a las nuevas regulaciones. Evidencias para politicas públicas en educación. Santiago: MINEDUC.

Bravo, D., Peirano, C., Sevilla, M. \& Wientraub, M. (200I). Informe ejecutivo del texto de investigación sobre el impacto de la formación dual en Chile. Santiago: Departamento de Economía de la Universidad de Chile.

Duk, C. \& Loren, C. (20I0). Flexibilización del currículum para atender a la diversidad. Revista Latinoamericana de Educación Inclusiva, I(4), 187-2I0.

Eitel, S. (2005). La integración escolar en Chile: perspectiva de los docentes sobre su implementación. Revista Iberoamericana sobre Calidad, Eficacia y Cambio en la Educación, 3 (I), 823-83I.

FONADIS (2004). Primer estudio de la discapacidad en Chile. Recuperado de http:/ / www.senadis.gob.cl/descarga/i/I29/documento

Garrido, J. \& Santana, R. (1994). Adaptaciones curriculares. Madrid: CEPE.

I El Campus Casona de Las Condes se encuentra ubicado en Fernández Concha 700, comuna de Las Condes. 
Gobierno de Chile (1994). Ley de Integración Social de las Personas con Discapacidad $N^{0}$ 19.284. Recuperado de https://www.ben.cl/

Izuzquiza, D. \& Rodríguez, P. (2010). Un análisis de resultados de la primera experiencia de formación en España para personas con discapacidad intelectual en el ámbito universitario. En Cabezas, D. \& Flórez, J., Educación postsecundaria en entorno universitario para alumnos con discapacidad intelectual: Experiencias y resultados (pp. 13-30). Madrid: Fundación Iberoamericana Down2I-Fundación Prodis.

Lissi, M., Zuzulich, M., Hojas, A., Achiardi, C., Salinas, S. \& Vásquez, A. (2013). En el camino bacia la educación superior inclusiva. Fundamentos $y$ adecuaciones curriculares para estudiantes con discapacidad sensorial o motora. Santiago: PUC.

Lucchini, G. (2003). Niños con necesidades educativas especiales: Cómo enfrentar el trabajo en el aula. Santiago: Ediciones Universidad Católica de Chile.

Marchesi, A. \& Coll, C. (1998). Desarrollo psicológico y educación. Madrid: Alianza.

Mertz, C. (200I). Políticas y programas para la prevención de la deserción escolar. Santiago: Fundación Paz Ciudadana.

MINEDUC (2004). Antecedentes bistóricos, presente y futuro de la Educación Especial en Chile. Recuperado de http://www.educacionespecial.mineduc.cl/

MINEDUC (2013). Orientaciones técnicas para programas de integración escolar (PIE). Recuperado de http://especial.mineduc.cl/implementaciondcto-supr-noI70/orientaciones/

Ministerio de Desarrollo Social (2015). Segundo estudio nacional de la discapacidad: Un nuevo enfoque para la inclusión. Recuperado de http://www. ministeriodesarrollosocial.gob.cl/pdf/ley-de-discapacidad.pdf

Moreno, M. (2005). Integración /inclusión de las personas con discapacidad en la Educación Superior. Caracas: IESALC.

Stainback, S., Stainback, W. \& Jackson, H. (1999). Hacia las aulas inclusivas. Madrid: Narcea.

SENADIS (2010). Ley N 20.422. Recuperado de http://www.senadis.gob.cl/ pag/I77/557/ley_n20422

UNESCO (1994). Informe final. Conferencia mundial sobre necesidades educativas especiales: Acceso y calidad. Madrid: UNESCO/Ministerio de Educación y Ciencia.

(2008). La educación inclusiva: El camino bacia el futuro. Ginebra: ONU. 
Verdugo, M. (2003). Análisis de la definición de discapacidad intelectual de la Asociación Americana sobre Retraso Mental de 2002. Revista Española sobre Discapacidad Intelectual, 34(205), 5-I9.

Vidal, R. \& Cornejo, C. (20I2). Empleo con apoyo, una estrategia de inserción laboral para jóvenes con discapacidad intelectual. Revista Convergencia Educativa, I27(I), II3-I27. 